\title{
ATENDIMENTO EM CLÍNICA DA FAMÍLIA: SIGNIFICADO DO CUIDADO PRESTADO POR ENFERMEIROS*
}

Juliana Nascimento Nogueira', Maria Helena do Nascimento Souza ${ }^{2}$

'Enfermeira. Especialista em Enfermagem deSaúdePública. SecretariaMunicipal deSaúdeeDefesa Civil do RiodeJaneiro. RiodeJaneiro-RJ-Brasil. ${ }^{2}$ Enfermeira. Doutora em Enfermagem. Universidade Federal do Rio de Janeiro. Rio de Janeiro-RJ-Brasil.

RESUMO: O estudo objetivou compreender os significados atribuídos pelo enfermeiro acerca do cuidado prestado em uma Clínica da Família. Trata-se de uma pesquisa exploratória qualitativa, embasada no referencial da fenomenologia sociológica de Alfred Schütz. Os participantes foram 10 enfermeiros de uma Clínica da Família do município do Rio de Janeiro. A coleta dos dados ocorreu mediante entrevista realizada no período de fevereiro a março de 2012. A partir da análise das entrevistas foi possível organizar os significados em três categorias: Acompanhar e cuidar da família, Promover a saúde e Satisfação profissional. Conclui-se que os significados do atendimento prestado perpassam a necessidade de considerar toda a família como foco da atenção, numa perspectiva de desenvolver ações voltadas para a promoção da saúde e melhoria da qualidade de vida da população, o que gera reconhecimento e autonomia profissional.

DESCRITORES: Programa saúde da família; Atenção primária à saúde; Enfermagem.

\section{CARE AT THE FAMILY CLINIC: MEANING OF CARE PROVIDED BY NURSES}

\begin{abstract}
The study aimed to understand the meanings assigned by nurses to the care provided in a Family Clinic. This is a qualitative exploratory study based on Alfred Schütz' social phenomenology. The participants were 10 nurses from a Family Clinic in the city of Rio de Janeiro. Data collection was carried out through interviews during the period from February to March 2012. Based on the analysis of the interviews, the meanings could be organized in three categories: Assisting and caring for family, Promoting health and Occupational satisfaction. We conclude that the meanings of care provided inspire the need to consider the whole family as the focus of care, with a view to developing initiatives to promote health and improve the quality of life of the population, which generates recognition and professional autonomy.
\end{abstract}

DESCRIPTORS: Family health program; Primary health care; Nursing.

\section{ATENDIMIENTO EN CLÍNICA DE LA FAMILIA: SIGNIFICADO DEL CUIDADO PRESTADO POR ENFERMEROS}

RESUMEN: El estudio tuvo la finalidad de comprender los significados señalados por el enfermero acerca del cuidado prestado en una Clínica de la Familia. Es una investigación exploratoria cualitativa, basada en el referencial de la fenomenología sociológica de Alfred Schütz. Los participantes fueron 10 enfermeros de una Clínica de la Familia del municipio de Rio de Janeiro. Los datos fueron obtenidos por medio de entrevista realizada en el periodo de febrero a marzo de 2012. Con base en los análisis de las entrevistas, fue posible organizar los significados en tres categorías: Acompañar y cuidar de la familia, Promover la salud y Satisfacción profesional. Se concluye que los significados del atendimiento prestado se relacionan a la necesidad de considerar toda la familia como foco de la atención, en una perspectiva de desarrollar acciones para la promoción de la salud y mejoría de la cualidad de vida de la población, lo que genera reconocimiento y autonomía profesional.

DESCRIPTORES: Programa Salud de la Familia; Atención primaria a la salud; Enfermería.

\footnotetext{
*Artigo extraído da Dissertação de Mestrado intitulada: "O enfermeiro na Clínica da Família: análise compreensiva da ação assistencial". Programa de Pós-graduação em Enfermagem da Escola de Enfermagem Anna Nery da Universidade Federal do Rio de Janeiro, 2012.
}

\section{Autor Correspondente:}

Maria Helena do Nascimento Souza

Universidade Federal do Rio de Janeiro

Rua Praia de Botafogo, 132 - 22250-040 - Rio de Janeiro-RJ-Brasil

E-mail:mhnsouza@yahoo.com.br
Recebido: $14 / 04 / 2014$ Finalizado: $23 / 10 / 2014$ 


\section{INTRODUÇÃO}

A Estratégia Saúde da Família (ESF) é entendida como uma forma de reorientação do modelo assistencial em saúde, operacionalizada em unidades básicas de saúde, que busca desenvolver atividades sob os princípios e diretrizes como adscrição dos usuários, integralidade, acessibilidade, variedade de serviços, continuidade da atenção, organização do trabalho fundamentado em uma equipe multiprofissional ${ }^{(1)}$.

As equipes de ESF são responsáveis pelo acompanhamento da situação de saúde das famílias residentes em uma área adscrita à unidade, mediante o planejamento, organização, desenvolvimento e avaliação de ações voltadas para a promoção da saúde, prevenção, recuperação e reabilitação de agravos que afetam a população, tendo a atenção primária como o referencial de seu trabalho ${ }^{(2-3)}$.

No processo de reorganização dos serviços, a atenção primária à saúde vem se consolidando e fortalecendo como a coordenadora do cuidado oferecido à população no sistema de saúde e como ordenadora do modelo assistencial fundamentado pela lógica das Redes de Atenção à Saúde, que visam racionalizar o uso de todos os recursos, tanto básicos quanto especializados, direcionados para a promoção, manutenção e melhoria da saúde ${ }^{(1,4-5)}$.

Um serviço de atenção primária à saúde dirigida à população pode ser considerado um provedor de cuidados primários quando apresenta, em seu processo de trabalho, atributos essenciais: acesso de primeiro contato do indivíduo com o sistema de saúde; longitudinalidade; integralidade e coordenação de atenção e; atributos derivados: orientação familiar; orientação comunitária e competência cultural. Tais atributos norteiam e qualificam as ações dos serviços de atenção primária à saúde, ampliando sua capacidade de interação com os indivíduos e com a comunidade $^{(4)}$.

No município do Rio de Janeiro, a ESF tem sido implementada com vistas a expandir o modelo assistencial da atenção primária à saúde, proposto no país. Para tanto, as ações voltadas para os cuidados primários de saúde são realizadas pelas equipes de ESF em unidades denominadas Clínicas da Família(6).
Em tais unidades de Saúde da Família destaca-se o papel do enfermeiro que tem a possibilidade de supervisionar e qualificar a equipe de enfermagem e os agentes comunitários de saúde, bem como realizar o co-gerenciamento da unidade. Nessa lógica, o enfermeiro passa a assumir importantes funções tanto no âmbito assistencial quanto administrativo, como:

educador, prestador de cuidados, consultor, auscultador/ identificador dos problemas da comunidade, articulador, integrador, planejador e interlocutor político ${ }^{(7: 381)}$.

Assim, neste modelo da atenção primária à saúde o cuidado de enfermagem deve ser voltado prioritariamente para ações de promoção da saúde e prevenção de agravos, com vistas à atenção integral à saúde da população atendida $^{(2,7-10)}$.

Justifica-se a relevância do presente estudo, uma vez que ele pode auxiliar na compreensão do significado da prática assistencial de enfermeiros no contexto da ESF, mais especificamente no cenário das Clínicas da Família. Nota-se que na literatura há uma lacuna no conhecimento acerca dos estudos fenomenológicos sobre o significado da prática assistencial no campo de atuação da Saúde da Família, sendo a maioria das investigações voltadas para a atuação de enfermeiros em áreas específicas como: saúde do idoso, saúde da mulher, saúde do adolescente e saúde da criança ${ }^{(11-13)}$.

Assim, este estudo poderá ampliar o olhar sobre a vivência deste profissional e o conhecimento dos motivos atribuídos por este em suas ações cotidianas, no cenário da Clínica da Família, contribuindo para a discussão dos modelos assistenciais a serem desenvolvidos pela Enfermagem na atenção primária à saúde.

Diante do exposto, considerando a atuação profissional do enfermeiro na área da saúde coletiva, mais especificamente na ESF, este estudo teve como objetivo compreender os significados atribuídos pelos enfermeiros, acerca do cuidado prestado em uma Clínica da Família.

\section{MÉTODO}

Trata-se de um estudo exploratório qualitativo em que se utilizou o referencial da fenomenologia sociológica de Alfred Schütz ${ }^{(14)}$. 
A opção pela fenomenologia social de Alfred Schütz se deu por esta possibilitar a adoção de um método sistemático para melhor compreensão dos aspectos sociais da ação humana dos enfermeiros que atuam em uma Clínica da Família. Essa abordagem fornece um caminho que articula conceitos de intersubjetividade, situações biográficas, relações sociais, motivações, expectativas e outros que possam melhor entender o mundo social das pessoas $^{(14)}$, e, nesse caso, o significado das ações intencionais dos enfermeiros, expresso na cotidianidade do seu contexto social de trabalho.

A pessoa em seu mundo da vida ao interagir com seus semelhantes estabelece uma relação intersubjetiva compartilhada, em que as experiências cotidianas, ações através das quais lida com seus interesses, manipulando objetos, tratando com pessoas, concebendo e realizando planos, são interpretadas reciprocamente ${ }^{(14)}$.

Neste mundo cotidiano o modo como a pessoa desenvolve suas ações e enfrenta seus desafios é definido pela sua situação biográfica, que corresponde à sedimentação de toda experiência vivida e do estoque de conhecimentos que esta tem à mão, sem os quais ela não pode interpretar suas experiências e observações, definir a situação em que se encontra e fazer planos ${ }^{(14)}$.

Para Alfred Schütz ${ }^{(14)}$ a conduta humana é considerada ação significativa, a partir do momento em que a pessoa age e atribui à ação um significado subjetivo. Para a compreensão desta ação humana faz-se necessário identificar a motivação que gerou um determinado comportamento social. Esta motivação pode ser expressa através de dois tipos de motivos: "motivos para", referentes às expectativas ou objetivos que se procura alcançar ou realizar, tendo em vista uma estrutura temporal voltada para o futuro e; "motivos porque", evidentes nos acontecimentos já concluídos, que explicam certos aspectos da realização do projeto tendo, portanto uma direção temporal voltada para as experiências passadas.

Com base nestes conceitos verificouse que a abordagem da fenomenologia sociológica constituiu em um caminho para a compreensão do significado da ação do enfermeiro que atua na Clínica da Família, uma vez que a prática do cuidado desenvolvido por este é uma ação social permeada de motivações, razões e objetivos profissionais.
O cenário foi uma Clínica da Família situada no município do Rio de Janeiro. Tal Clínica possuía onze equipes de Saúde da Família e três equipes de Saúde Bucal. As equipes eram responsáveis por uma população cadastrada no total de 34.900 pessoas aproximadamente o equivalente a 12.900 famílias, cerca de $79 \%$ do total da população estimada levando em consideração que a população total da área de adscrição da Clínica da Família pesquisada era de 44.000 habitantes $^{(6)}$.

Como critério de inclusão considerou-se os enfermeiros que estavam exercendo sua ação assistencial em uma das equipes da Clínica da Família, cenário do estudo, independente do tempo de formação e contratação. Foi excluído o enfermeiro responsável pela gerência da Unidade que não desenvolvia a prática assistencial junto aos usuários. Também, foi excluída a equipe em que um dos pesquisadores atuava como enfermeira.

A coleta dos dados ocorreu mediante uma entrevista fenomenológica realizada no período de fevereiro a março de 2012, em que se utilizou um roteiro composto por dados de caracterização dos sujeitos e pela seguinte questão: $O$ que significa para você prestar atendimento na Clínica da Família?

Os enfermeiros foram convidados a participarem do estudo após a explicação do objetivo e assinatura do Termo de Consentimento Livre e Esclarecido, em atenção à Resolução 196/96 do Conselho Nacional de Saúde ${ }^{(15)}$. Foi solicitada, ainda, a autorização para a gravação da entrevista em um mini player (MP3) e assegurado o anonimato dos participantes. As falas foram identificadas pela letra E (Enfermeiro) seguidas de números arábicos, conforme a ordem em que as entrevistas foram realizadas.

Para a interpretação dos resultados, após a transcrição dos depoimentos obtidos foi realizada leitura e releitura do material com a finalidade de captar a repetição dos aspectos comuns das falas dos participantes do estudo. A partir da apreensão de tais aspectos, emergiram as categorias concretas do vivido que desvelaram os significados atribuídos pelos enfermeiros em sua ação assistencial. O processo de categorização e a análise compreensiva destes significados foram realizados de acordo com o referencial metodológico da fenomenologia social de Alfred Schütz ${ }^{(14)}$. 
Esta pesquisa foi aprovada pelo Comitê de Ética em Pesquisa da Escola de Enfermagem Anna Nery - HESFA/UFRJ, sob o registro $n^{\circ}$ 100/2011 e autorizada pelo responsável técnico da unidade onde a pesquisa foi realizada.

\section{RESULTADOS}

Os dados de caracterização mostram que dentre os dez enfermeiros entrevistados dois eram do sexo masculino, oito do sexo feminino. Estes possuíam a idade média equivalente a 30,7 anos, variando de 23 a 48 anos; o tempo mediano de formação correspondente a 4 anos, variando de 1 a 20 anos; o tempo mediano de atuação na Clínica da Família de 8 meses, variando entre 1 a 24 meses e todos possuíam pelo menos um Curso de Especialização, sendo a maioria na área da Saúde Pública.

A partir da análise das informações obtidas nas entrevistas, em resposta ao questionamento: "O que significa para você prestar atendimento na Clínica da Família?", foi possível organizar os significados em três categorias: Acompanhar e cuidar da família, Promover a saúde e Satisfação profissional.

Na categoria Acompanhar e cuidar da família, a análise dos depoimentos desvelou que os enfermeiros em sua prática assistencial procuram: desenvolver ações de promoção e prevenção da saúde, realizar o cuidado ao indivíduo e sua família em todo o ciclo de vida identificando os processos sociais que interferem em sua saúde, como evidenciado nas falas a seguir:

[...] a gente entra dentro da casa, participa, consegue estar junto da família, entender os problemas, atender desde a criança até o idoso, então consegue ter uma abordagem melhor da população, [...] ter o cuidado, conhecer toda aquela família, todas as gerações. (E01)

Acompanhar tanto o indivíduo como a sua família, orientando e evitando possíveis doenças e agravos. (E03)

Significa conhecer melhor as pessoas que você está atendendo, significa chamar estas pessoas para construir o atendimento delas, ter uma proposta de trabalhar com o território, entender que os fatores sociais interferem na qualidade de vida e na saúde dessa população. (E04)

É um acompanhamento do usuário, da família. (E05)

De outra forma, a categoria Promover a saúde mostra que o significado atribuído pelos enfermeiros que atuam na Clínica da Família está focalizado no fato de realizar atividades preventivas e de promoção da saúde, a fim de melhorar a qualidade de vida da população:

Acho que a gente consegue mudar os hábitos de vida, [...] ter uma abordagem melhor da população, não só aquela visão hospitalar voltada para doença. (E01)

Significa estar desenvolvendo ações [...] assistenciais, de promoção, de prevenção [...]. (E02)

Significa promover a saúde, melhorar a qualidade de vida da população da região [...] hoje em dia com a Clínica da Família a qualidade de saúde melhorou muito. (E07)

Quando questionados sobre o significado da atuação profissional no ambiente da Clínica da Família os enfermeiros, na categoria Satisfação profissional mencionaram a importância de realizar ações voltadas para um atendimento diferenciado à clientela, que geram satisfação e reconhecimento do valor da profissão. Ao mesmo tempo referiram que atuar na ESF significa autonomia profissional, como evidenciado nas falas:

É um atendimento diferenciado que a gente faz. (E05)

Poder levar os meus conhecimentos o mais direitamente possível às pessoas [...]. (E06)

Poder exercer de melhor maneira a enfermagem de uma forma mais autônoma [...] na Clínica da Família eu acho que o enfermeiro consegue ser mais autônomo. (E08) 
Significa uma satisfação, porque a gente tem certo reconhecimento de alguns pacientes, a gente vê que o nosso trabalho é bem aceito, os pacientes gostam e elogiam. (E10)

\section{DISCUSSÃO}

O conhecimento dos significados atribuídos pelos enfermeiros, com relação a sua atuação no cenário de Clínica da Família, possibilitou apreender o sentido da sua ação assistencial. Segundo o referencial teórico deste estudo foi possível entender que o viver no mundo da vida cotidiana significa viver em um envolvimento interativo com muitas pessoas, em complexas redes de relacionamentos sociais. Nestas redes as pessoas podem estabelecer relações do tipo face a face, que pressupõe orientação voltada para o outro, considerando este ser humano como pessoa que tem suas vivências e experiências ${ }^{(14)}$.

A categoria Acompanhar e cuidar da família desvela, por parte do enfermeiro, a preocupação em estabelecer relação de proximidade com o usuário possibilitando a identificação das demandas de saúde e o acompanhamento das pessoas atendidas, tendo em vista o desenvolvimento de ações de promoção da saúde.

Estes resultados estão de acordo com estudo que mostra que na prática de atenção à saúde o enfermeiro tem assumido um papel decisivo e pró-ativo no que se refere à identificação das necessidades de cuidado da população, bem como na promoção e proteção da saúde dos indivíduos em suas diferentes dimensões ${ }^{(16)}$. Isto demanda o aprimoramento de suas competências para decidir, viabilizar intervenções compatíveis com aspectos ético-legais, que atendam as necessidades dos indivíduos, famílias e comunidade ${ }^{(17)}$.

No que tange ao acompanhamento à saúde das famílias, o enfermeiro tem caráter mais generalista do que de especialista e muitas vezes, deve ter capacidade para realizar inúmeras tarefas, trabalhar em equipe e apresentar raciocínio rápido para agir com competência, paciência, segurança e persistência em diversas circunstâncias ${ }^{(8)}$. Entretanto, estudo mostra que embora a ESF objetive a reorganização da prática assistencial na atenção básica, a ação dos profissionais ainda é baseada na lógica do modelo de atenção centrado na doença e na assistência individual $^{(18)}$.
De acordo com Schütz ${ }^{(14)}$ o mundo da vida cotidiana não é de forma alguma um mundo privado ou individual, mas é desde o início, um mundo intersubjetivo compartilhado entre semelhantes, vivenciado e interpretado pelas pessoas; em suma é um mundo comum a todos.

Na categoria Promover a Saúde, os enfermeiros participantes deste estudo observaram a relevância das ações de promoção da saúde, que sendo uma estratégia articulada com as demais políticas é de extrema importância para o desenvolvimento de intervenções que possam causar impacto na saúde dos indivíduos e da comunidade ${ }^{(9)}$.

Ao realizarem as intervenções em saúde os enfermeiros tem como base a bagagem ou estoque de conhecimentos acumulados ao longo da vida, que caracterizam a situação biográfica e influenciam a sua forma de interpretação do mundo ${ }^{(14)}$.

Os achados de uma pesquisa realizada no sul do país, sobre o significado da atuação de enfermeiros em uma equipe de ESF, mostram que o comprometimento do enfermeiro com a comunidade, mediante ações de promoção da saúde e prevenção de doenças, tem contribuído para a melhoria da qualidade de vida dos indivíduos e famílias ${ }^{(11)}$.

Os dados também estão de acordo com um estudo de revisão integrativa que mostrou que quando o enfermeiro utiliza o conceito de promoção da saúde em sua prática assistencial, muitos resultados positivos à saúde podem ser obtidos como: adesão, qualidade de vida, o conhecimento dos pacientes sobre suas doenças e o auto-cuidado ${ }^{(19)}$. No entanto, embora a Enfermagem mostre reconhecer a importância da Promoção da Saúde em sua ação assistencial, ainda muitas atividades do enfermeiro estão limitadas em orientações de boas práticas de saúde e outros aconselhamentos afins ${ }^{(20)}$.

Enfim, na categoria Satisfação profissional, os enfermeiros evidenciaram ainda a possibilidade de realizarem ações que trazem satisfação, autonomia e reconhecimento profissional. Tais achados corroboram com estudos que destacam as percepções de valorização, satisfação, identificação com a área da atenção básica, recompensa salarial, reconhecimento do seu desempenho e maior autonomia profissional, como determinantes para a motivação dos enfermeiros no trabalho da $\operatorname{ESF}^{(1-3,12-13)}$. 
Os motivos que permeiam o significado da ação assistencial prestada pelos enfermeiros, como sujeitos de sua história e ponto de referência para a equipe da ESF, possibilitaram a discussão sobre o papel destes profissionais e sobre os avanços e dificuldades presentes nesta Estratégia $^{(21-22)}$.

Desta forma, com base na fenomenologia social de Alfred Schütz ${ }^{(14)}$, o cuidado de enfermagem em uma Clinica da Família pode ser considerado como uma ação social, que o enfermeiro desenvolve no mundo da vida cotidiana onde estabelece diversas relações intersubjetivas. Ao agir neste contexto social o enfermeiro busca valorizar as ações de promoção da saúde utilizando o estoque de conhecimentos, as experiências adquiridas ao longo da vida, bem como a situação biográfica em que se encontra no momento do cuidado prestado.

Nesta perspectiva, ressalta-se a importância do trabalho de aproximação dos enfermeiros com as famílias atendidas, mediante uma relação face a face ${ }^{(14)}$ de respeito e o estabelecimento de um vínculo de confiança, a fim de promover assistência de qualidade voltada para as reais necessidades de saúde dos usuários.

\section{CONCLUSÃO}

Para os enfermeiros, os significados do atendimento prestado na Clínica da Família perpassam pela necessidade de considerar toda a família como foco da atenção, numa perspectiva de desenvolver ações voltadas para a promoção da saúde e melhoria da qualidade de vida da população, o que gera reconhecimento e autonomia profissional.

Esta investigação se limitou à vivência de um determinado grupo de enfermeiros, situado em um dado contexto sócio-histórico-cultural, o que impede a generalização dos resultados. No entanto, os achados permitiram compreender que os significados atribuídos pelos enfermeiros neste estudo são consoantes com as recomendações governamentais no que tange ao desenvolvimento das ações de promoção da saúde no âmbito da Estratégia Saúde da Família.

Nesta perspectiva, este estudo contribui para reflexão sobre o cuidado neste modelo de atenção à saúde, constituindo em subsídio para a avaliação e reorientação das práticas assistenciais desenvolvidas pelo enfermeiro, sujeito protagonista das ações de saúde voltadas para o bem estar da comunidade atendida.

\section{REFERÊNCIAS}

1. Starfield B. Primary care: an increasingly important contributor to effectiveness, equity, and efficiency of health services SESPAS report 2012. Gac. Sanit. 2012; 26 Suppl 1: 20-6.

2. Ministério da Saúde (BR). Portaria GM no. 2488, de 21 de outubro de 2011. Aprova a Política Nacional de Atenção Básica, estabelecendo a revisão de diretrizes e normas para a organização da Atenção Básica, para a Estratégia Saúde da Família (ESF) e o Programa de Agentes Comunitários de Saúde (PACS). Diário Oficial da União. Brasília; 2011.

3. Ribeiro LCC, Ribeiro M, Dias KS, Matos KA, Ferreira TS. O diagnóstico administrativo e situacional como instrumento para o planejamento de ações na estratégia saúde da família. Cogitare enferm. 2008;13(3):448-52.

4. Ministério da Saúde (BR). Política Nacional de Promoção da Saúde. $3^{\text {a }}$ edição, Séries Pacto pela Saúde. Brasília; 2010. 2010. 59p.

5. Mendes EV. As redes de atenção à saúde. Brasília: Organização Pan-Americana da Saúde; 2011. 554 p.

6. Secretaria Municipal de Saúde e Defesa Civil. Clínicas da Família: a SMSDC-RJ inovando na atenção à saúde dos cariocas. Série Mais Saúde Cidade do Rio de Janeiro. [Internet]. 2010 [acesso em 10 de junho de 2012]. Disponível em: http://voticscxs1.otics.org/ trabalhosredeunida/resumos/RE1539-1.pdf

7. Aguiar AC, Barbosa SP. Fatores influentes na permanência dos enfermeiros na estratégia de saúde da família em Ipatinga. Rev. APS. 2008;11(4):380-8.

8. Figueiredo NMA, Tonini T, organizadores. SUS e PSF para enfermagem: práticas para o cuidado em Saúde Coletiva. $1^{\text {a }}$ ed. São Paulo: Yends; 2008.

9. Araújo MFS, Oliveira FMC. A atuação do enfermeiro na equipe de saúde da família e a satisfação profissional. [Internet]. Rev. Eletrônica Ciênc Soc. 2009;1(14) [acesso em 19 ago 2012] Disponível: http://paginas. cchla.ufpb.br/caos/n14/DOSSIE\%20SA\%C3\%9ADE_ TEXTO\%20I_ATUA\%C3\%87\%C3\%83O\%20DO $\% 20$ ENFERMEIRO.pdf

10. Saito RXS. Integralidade da atenção: organização do trabalho no programa saúde da família na perspectiva sujeito-sujeito. São Paulo: Martinari; 2008. 
11. Chrizostimo MM, Rosas AMMTF, Alves L, Bartoly MG, Silva CMC, Alves EMC. O significado da assistência de enfermagem segundo abordagem de Alfred Schütz. Cienc. enferm. 2009; 15(3):21-8.

12. Torres CA, Popim RC, Carregã K, Spiri WC. Integralidade do cuidado com o idoso na estratégia de saúde da família: visão da equipe. Esc. Anna Nery. 2013; 17(4): 628-37.

13. Oliveira FFS, Oliveira ASS, Lima LHO, Marques MB, Felipe GF, Sena IVO. Consulta de puericultura realizada pelo enfermeiro na estratégia saúde da família. Rev. Rene. 2013; 14(4):694-703.

14. Schütz A. Sobre fenomenologia e relações sociais. Petrópolis (RJ): Vozes; 2012.

15. Ministério da Saúde (BR). Conselho Nacional de Saúde. Diretrizes e normas regulamentadoras sobre pesquisa envolvendo seres humanos. Resolução 196, de 10 de outubro de 1996. Brasília; 1996.

16. Backes DS, Backes MS, Erdmann AL, Büscher A. O papel profissional do enfermeiro no Sistema Único de Saúde: da saúde comunitária à estratégia de saúde da família. Ciênc. saúde coletiva. 2012;17(1):223-30.

17. Costa LB, Saraiva MRB, Barroso MGT. Aplicações de estruturas conceituais na consulta de enfermagem à família. Esc. Anna Nery. 2011;11(3):515-9.

18. Arreaza ALV, Moraes JC. Vigilância da saúde: fundamentos, interfaces e tendências. Cienc. saude colet. 2010;15(4):2215-28.

19. Kemppainen V, Tossavainen K, Turunen H. Nurses' roles in health promotion practice: an integrative review. Health promot. int. 2013;28:490-501.

20. Whitehead D. Health promotion in nursing: a derridean discourse analysis. Health promot. int. 2011;26:117-27.

21. Rangel RF, Fugali MM, Backes DS, Gehlen $M H$, Souza MHT. Avanços e perspectivas da atuação do enfermeiro em estratégia saúde da família. Cogitare enferm. 2011;6(3):498-504.

22. Souza MG, Mandú ENT. Percepções de enfermeiros sobre a estratégia saúde da família. Ciência, Cuidado e Saúde. 2010;9(4):643-50. 